TITLE:

\title{
Effects of two stretching methods on shoulder range of motion and muscle stiffness in baseball players with posterior shoulder tightness: a randomized controlled trial
}

\section{$\operatorname{AUTHOR}(\mathrm{S})$ :}

Yamauchi, Taishi; Hasegawa, Satoshi; Nakamura, Masatoshi; Nishishita, Satoru; Yanase, Ko; Fujita, Kosuke; Umehara, Jun; Ji, Xiang; Ibuki, Satoko; Ichihashi, Noriaki

\section{CITATION:}

Yamauchi, Taishi ... [et al]. Effects of two stretching methods on shoulder range of motion and muscle stiffness in baseball players with posterior shoulder tightness: a randomized controlled trial. Journal of shoulder and elbow surgery 2016, 25(9): 1395-1403

\section{ISSUE DATE:}

2016-09

URL:

http://hdl.handle.net/2433/231304

\section{RIGHT:}

(C) 2016. This manuscript version is made available under the CC-BY-NC-ND 4.0 license

http://creativecommons.org/licenses/by-nc-nd/4.0/. The full-text file will be made open to the public on 01 September 2017 in accordance with publisher's 'Terms and Conditions for Self-Archiving'. This is not the published version. Please cite only the published version. この論文は出版社版でありません。引用の際には出版社版をご確認ご利用ください。 
Posterior shoulder stretching in baseball players

$7 \quad$ Taishi Yamauchi, PT, MSc ${ }^{1}$

8 Satoshi Hasegawa, PT, $\mathrm{PhD}^{1}$

9 Masatoshi Nakamura, PT, $\mathrm{PhD}^{1,2}$

10 Satoru Nishishita, PT, MSc ${ }^{1}$

11 Ko Yanase, $\mathrm{PT}^{1}$

$12 \quad$ Kosuke Fujita, $\mathrm{PT}^{1}$

13 Jun Umehara, $\mathrm{PT}^{1}$

$14 \quad$ Xiang $\mathrm{Ji}^{1}$

15 Satoko Ibuki, PT ${ }^{1}$

16 Noriaki Ichihashi, PT, $\mathrm{PhD}^{1}$ 
Posterior shoulder stretching in baseball players

18

19

20

21

22

23

24

25

26

27

28

29

30

31

32

33

34

1Human Health Sciences, Graduate School of Medicine, Kyoto University, Kyoto, Japan

2Faculty of Health and Sports Science, Doshisha University, Kyoto, Japan

*Corresponding author: Taishi Yamauchi, PT, MSc

Human Health Sciences, Graduate School of Medicine, Kyoto University

53 Shogoin-Kawahara-cho, Sakyo-ku, Kyoto 606-8507, Japan

Phone: +81-75-751-3935; Fax: +81-75-751-3909

E-mail: h2sutofo@gmail.com

\section{Disclaimer:}

This work was supported by the Grant-in-Aid for Scientific Research (B) 15H03043. The funding source played no role in the design, implementation, or data analysis, interpretation, or reporting of the study. The authors, their immediate families, and any research foundations with which they are affiliated have not received any financial payments or other benefits from any commercial entity related to the subject of this article.

This study has been approved by the Ethics Committee of the Kyoto University Graduate School and Faculty of Medicine (approval no.: E2331). 
Posterior shoulder stretching in baseball players

36

\section{Abstract}

Background: The cross-body stretch and sleeper stretch are widely used for improving flexibility of the posterior shoulder. These stretching methods were modified by Wilk. However, few quantitative data are available on the new, modified stretching methods. A recent study reported the immediate effects of stretching and soft tissue mobilization on the shoulder range of motion (ROM) and muscle stiffness in subjects with posterior shoulder tightness. However, the long-term effect of stretching for muscle stiffness is unknown. The objective of this study is to examine the effects of two stretching methods, the modified crossbody stretch (MCS) and the modified sleeper stretch (MSS), on shoulder ROM and muscle stiffness in baseball players with posterior shoulder tightness.

Methods: Twenty-four college baseball players with ROM limitations in shoulder internal rotation were randomly assigned to the MCS or MSS group. We measured shoulder internal rotation and horizontal adduction ROM and assessed posterior shoulder muscle stiffness with ultrasonic shear wave elastography before and after a 4-week intervention. Subjects were asked to perform 3 repetitions of the stretching exercises every day, for $30 \mathrm{~s}$, with their dominant shoulder.

Results: In both groups, shoulder internal rotation and horizontal adduction ROM were significantly increased after the 4-week intervention. Muscle stiffness of the teres minor decreased in the MCS group and that of infraspinatus decreased in the MSS group.

Conclusions: The MCS and MSS are effective for increasing shoulder internal rotation and horizontal adduction ROM and improving muscle stiffness of the infraspinatus or teres minor. 
Posterior shoulder stretching in baseball players

57

58 Level of evidence: Treatment study, randomized controlled study, level 2

59

60 Key words:

61 shear wave elastography; modified sleeper stretching; modified cross-over stretching;

62 posterior shoulder tightness; baseball; infraspinatus; teres minor

63

64 
Posterior shoulder stretching in baseball players

65

66

67

68

69

70

71

72

73

74

75

6

\section{Introduction}

In the throwing motion in baseball, significant force is generated in the posterior shoulder, especially in the release to follow-through phases ${ }^{10}$. Due to this force generation, baseball players often exhibit glenohumeral internal rotation deficit (GIRD) and glenohumeral horizontal adduction deficit (GHAD) in their throwing $\operatorname{arm}^{3,4}$, 25, 34, 35 . Limitation in range of motion (ROM) may be caused by reduced soft tissue flexibility in the posterior shoulder region, referred to as posterior shoulder tightness ${ }^{4,25}$. Baseball players

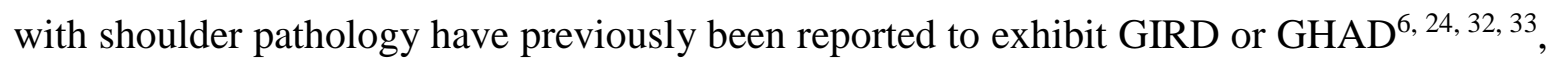
and those with GIRD or GHAD have been reported to be at high risk for developing shoulder pathology ${ }^{34,38}$; posterior shoulder tightness is therefore considered to be related to throwing injuries.

In regard to the relationship between posterior shoulder tightness and soft tissues in the posterior shoulder region, several studies have focused on the posterior glenohumeral joint capsule $\mathrm{e}^{11-13,22,23,35}$. On the other hand, several other studies have correlated certain muscles and posterior shoulder tightness, with some of them suggesting that baseball pitching and exercises involving shoulder external rotators are associated with immediate development of GIRD or GHAD along with exhaustion or mobility deficits of shoulder external rotators $8,28,31,40$. In addition, some reports have shown increase in shoulder internal rotation (IR) or horizontal adduction (HA) ROM with physical therapy aimed at improving extensibility of the posterior shoulder muscles $2,4,21,30,41$ or with dissection of the infraspinatus and teres minor muscles in cadaveric shoulders ${ }^{5}$. A recent study by Bailey et al. showed that the decrease of the infraspinatus stiffness leads to acute gain in shoulder ROM². 
Posterior shoulder stretching in baseball players

87 Therefore, not only the posterior glenohumeral joint capsule, but also the posterior shoulder

the differences in muscle stiffness between the throwing and non-throwing sides ${ }^{2}$.

Among the various stretching methods developed with the aim of reducing posterior shoulder tightness, the cross-body stretch, in which the shoulder is horizontally adducted, and the sleeper stretch, in which the shoulder is internally rotated, are used widely ${ }^{17-20,27}$. Recently, a few authors proposed that scapular stabilization during the cross-body stretch enhanced the stretching effects on the posterior glenohumeral joint ${ }^{27,38}$. Indeed, Salamh et al. demonstrated that manual scapular stabilization increases the effects of stretching, when the shoulder is horizontally adducted by a therapist ${ }^{33}$. On the other hand, these stretching methods can be painful in some cases ${ }^{20}$. For these reasons, Wilk et al. developed the modified cross-body stretch (MCS) and the modified sleeper stretch (MSS) ${ }^{38}$. However, little is known about the effects of these stretching methods for reducing GIRD and GHAD. In addition, the effects of these stretching exercises on muscle stiffness, which can be measured as shear elastic modulus using ultrasonic shear wave elastography (SWE) imaging ${ }^{26}$, are not clear.

Therefore, this study aimed to compare baseline glenohumeral ROM and muscle stiffness between the throwing and non-throwing sides and to examine the effects of an intervention using the MCS and MSS in baseball players with posterior shoulder tightness of the throwing side. This information will help clinicians select the appropriate stretching method for preventing and improving posterior shoulder tightness in baseball players. 
Posterior shoulder stretching in baseball players

108

111

112

114

116

117

118

119

\section{Materials and Methods}

This is a randomized controlled study examining the effects of the MCS and MSS performed for 4 weeks in college baseball players with posterior shoulder tightness.

\section{Subjects}

Twenty-four college baseball players volunteered for this study. They were randomly assigned to the MCS $(\mathrm{N}=12)$ or MSS groups $(\mathrm{N}=12)$. The inclusion criterion for selection of players that they were participating in daily practice, had posterior shoulder tightness which was evaluated as the presence of GIRD $>10^{\circ}$ on the throwing side compared with the non-throwing side $\mathrm{e}^{20,29}$. The exclusion criterion was inability to perform stretching exercises because of injury or pain, a history of surgery of the upper arm, or being rehabilitated for the disabled throwing shoulder. Using previously published changes in muscle shear elastic modulus after stretching intervention ${ }^{26}$, a power of 0.80 , an alpha level of 0.05 , and large f of 0.4 were assumed for the two-way factorial analysis of variance, which determined the sample size of 13 per group. Those who were injured during the intervention and were unable to perform stretching exercises were excluded from the analysis. Written informed consent was obtained from each participant. This study was approved by the ethics committee of the Kyoto University Graduate School and Faculty of Medicine (approval number E2331).

\section{Procedures}


Posterior shoulder stretching in baseball players

The testing was conducted in a laboratory at the Kyoto University. Twenty-four participants were randomized by the author using computer-generated permuted block randomization. The permutation lists were CCSS, CSCS, CSSC, SSCC, SCSC, and SCCS (C: MCS, S: MSS). A series randomization procedure was conducted after the recruitment. All measurements were performed by one tester with one or two assistants, who were not blinded to the group assignment. Bilateral pre- and post-intervention (4 weeks) glenohumeral ROM and muscle stiffness were assessed in each subject. To reduce deterioration of reproducibility, the pre- and post-intervention measurements were performed at the same time of the day.

\section{Glenohumeral ROM Measurements}

Prior to the ROM measurement, the subjects performed warm-up exercises consisting of 3 repetitions of shoulder flexion, held at the end range with hands clasped, for $10 \mathrm{~s}^{20}$. We used a digital angle meter (WR300, Wixey, USA) to measure passive glenohumeral IR, external rotation (ER), and horizontal adduction (HA) ROM. The ROM measurement method conformed to that used in previous studies ${ }^{37,39}$. ROM measurements were performed with subjects in the supine position, the test shoulder in $90^{\circ}$ abduction and elbow in $90^{\circ}$ flexion, and the scapula stabilized. Each measurement was performed twice, and the average values were used for analysis. Total ROM was calculated by adding the IR and ER ROM.

\section{Assessment of Shoulder Muscle Stiffness Using SWE}


Posterior shoulder stretching in baseball players

150

151

152

153

154

155

156

157

158

159

160

161

162

163

164

165

166

167

168

169

170

171

We used the ultrasonic SWE with a 2-10 MHz linear array probe (Aixplorer, SuperSonic Imagine, Aix en Provence, France) to assess stiffness (shear elastic modulus) of the posterior shoulder muscles, i.e., infraspinatus, teres minor, and posterior deltoid. The previous study reported that the muscle shear modulus measured by using the ultrasonic SWE is highly correlated with Young's modulus from traditional material testing ${ }^{9}$. The ultrasonic SWE could measure the muscle shear modulus at a wide range, and it has high repeatability, with values of 0.978 and 0.948 between trials and between days, respectively ${ }^{42}$. In the assessment using SWE, a color-coded box showing the shear elastic modulus was superimposed on the B-mode ultrasound image, and the circular region of interest was set near the central part of the muscle ${ }^{26}$ (Fig. 1). In this study, we used the average circular region of interest for analysis.

Assessment of muscle stiffness was performed in two positions: (1) the subject in the sitting position, with the test shoulder in $90^{\circ}$ abduction and $40^{\circ} \mathrm{IR}$, and the elbow in $90^{\circ}$ flexion (2nd IR); (2) the subject in the sitting position, with the test shoulder in $110^{\circ} \mathrm{HA}$ and the elbow in $90^{\circ}$ flexion (HA). Subjects were instructed to remain relaxed, and their shoulder was moved passively to the assessment position by an assistant. The shoulder and elbow angles were confirmed with a goniometer, and the assistant supported the arm during stiffness measurement. For the measurement at the 2nd IR position, the scapula was stabilized by another assistant who grasped the coracoid. However, the scapula was not stabilized during the measurement in the HA position because the probe placement was near the lateral border of the scapula, which could not be grasped for stabilization. The probe placement for each muscle was as follows (Fig.2): The infraspinatus was measured at the midpoint between the 
Posterior shoulder stretching in baseball players

172 spine of the scapula and inferior angle of the scapula, and the probe was placed parallel to

173 the infraspinatus. The teres minor was measured near the midpoint of the inferior angle of

174 the scapula and the greater tubercle, where the teres minor was identified with the probe

175 vertical to it; the probe was then placed parallel to the teres minor. The posterior deltoid was

176 measured $4 \mathrm{~cm}$ below the posterior acromion. Each measurement was performed twice, and

177 the average of the two values was used for analysis.

\section{Two Stretching Methods - MCS and MSS}

The modified conventional stretching methods, i.e., the MCS and MSS, are shown in

Fig.3. The MCS was performed with the subjects in the side lying position on the throwing side to stabilize the scapula; the forearms were aligned, with the opposite forearm on top to restrict external rotation of the stretched shoulder; and the humerus of the throwing side was moved into HA using the opposite arm. The MSS was performed with the subjects in the side lying position on the throwing side; the trunk was rolled $30^{\circ}$ posteriorly on the throwing side to decrease the pressure at the glenohumeral joint; a towel was placed under the subject's humerus to increase the amount of glenohumeral HA; and the humerus of the throwing side was moved into IR using the opposite arm. Subjects were instructed to perform 3 repetitions

189 of the stretches on the throwing side only, once daily after practice or before going to bed, 190 for 4 weeks, and to hold each stretch for 30 s. 
Posterior shoulder stretching in baseball players

193

194

Because no intervention was applied to the non-throwing side, the intra-rater reliability of each measurement was established using the pre- and post- intervention values of the nonthrowing side. The average value of two measurements was used for calculating the intraclass correlation coefficient [ICC $(1,2)]$. The ICC $(1,2)$ values for each measurement are shown in Table 1. The standard error of mean (SEM) values of each item are also shown in the same table. In regard to the intra-rater reliability in this study, the ICC $(1,2)$ values for glenohumeral ROM and muscle stiffness were $>0.8$ and $>0.7$, respectively. Landis and Koch proposed that ICC values from 0.61 to 0.80 should be considered as "good" and those from 0.81 to 1.00 as "very good"

\section{Statistical Analysis}

R 2.8.1 was used to provide the ICC $(1,2)$ and the SEM. SPSS ver. 17 (SPSS Japan, Tokyo, Japan) was used for statistical processing. To compare the baseline glenohumeral ROM and muscle stiffness between the throwing and non-throwing sides, we used the paired t-test or Wilcoxon signed-rank test depending on whether the data followed a normal distribution. To examine the effect of intervention with respect to all variables, a two-way factorial analysis of variance (group $\times$ time) was used, and post hoc comparison was made for the main effect using the paired t-test or Wilcoxon signed-rank test depending on whether the data followed a normal distribution. Effect sizes were calculated using Microsoft Excel. Between the throwing and non-throwing sides, the effect size was calculated as [throwing side mean - non-throwing side mean]/pooled SD, and within-group effect size was 
Posterior shoulder stretching in baseball players

214 calculated as [post mean - pre mean]/pre SD. Differences were considered statistically

215 significant at values of $\mathrm{P}<0.05$

216 
Posterior shoulder stretching in baseball players

217

218

220

221

222

223

224

225

226

227

Subjects were recruited from July 26 to November 15, 2014. In expectation of losses to follow up, we recruited 24 subjects overall. One of the subjects in the MSS group was excluded from the analysis due to an injury experienced during baseball practice involving the non-throwing shoulder, following which he was unable to continue with the stretching intervention. As a result, we analyzed 12 and 11 subjects in the MCS and MSS groups, respectively, who completed this study protocol (Fig. 4). We verbally confirmed that the subjects have performed the stretching more than $70 \%$ of days during the intervention period. No significant differences were found between the two groups at baseline (Table 2).

\section{Comparison of Dominant and Non-dominant Shoulders}

The baseline glenohumeral ROM and muscle stiffness for the throwing and nonthrowing sides are shown in Table 3. The IR and HA ROM were smaller, and the ER ROM was larger on the throwing side compared with the non-throwing side $(\mathrm{P}<0.01)$. In regard to muscle stiffness, the infraspinatus and teres minor at the 2nd IR position and the teres minor at the HA position had greater muscle stiffness on the throwing side than those on the non-throwing side $(\mathrm{P}<0.01)$. The posterior deltoid showed no significant differences between the throwing and non-throwing sides.

\section{Shoulder ROM}


Posterior shoulder stretching in baseball players

239 IR and HA ROM, but no interaction effects were found between groups. As a result of post

240 hoc comparison in both groups, the IR ROM (both groups; P < 0.01) and HA ROM (MCS; P

$241<0.01$, MSS; $\mathrm{P}<0.05)$ were increased.

\section{Shoulder Muscle Stiffness}

The effects of 4 weeks of stretching on muscle stiffness are shown in Table 5. A

245 significant main effect difference was found for time on the infraspinatus and teres minor at

246 both positions, but no interaction effects were found between groups. As a result of post hoc

247 comparison, muscle stiffness of the teres minor was decreased at both positions in the MCS

248 group (both positions; $\mathrm{P}<0.05$ ). In the MSS group, muscle stiffness of the infraspinatus was

249 decreased at both positions (2nd IR; $\mathrm{P}<0.01$, HA; $\mathrm{P}<0.05$ ). No significant main effect were 250 found on the posterior deltoid. 
Posterior shoulder stretching in baseball players

252

\section{Discussion}

This study examined the effects of 4 weeks of the MCS and MSS in baseball players with posterior shoulder tightness of the glenohumeral joint and muscle stiffness. First, we compared the baseline glenohumeral ROM and muscle stiffness between the throwing and non-throwing sides. In similar previous studies, IR ROM and HA ROM were smaller, and ER ROM was larger on the throwing side compared with the non-throwing $\operatorname{side}^{2-4,25}, 35,36$. In regard to muscle stiffness, the infraspinatus and teres minor showed significantly greater stiffness on the throwing side than the non-throwing side. In the previous study examining shoulder muscle stiffness using SWE, no difference was found between the throwing and the non-throwing sides in the stiffness of the infraspinatus ${ }^{2}$. This finding is not in accordance with our results. This discrepancy may be due to the difference in the subject's measurement position and the measured region. Some of the previous studies have reported that an immediate decrease in glenohumeral IR and HA ROM was induced with baseball pitching or exercises involving shoulder external rotators together with exhaustion or mobility deficits of these muscles ${ }^{7,28,31,39}$. In prior research using SWE, muscle stiffness increased immediately after exercises, thereby causing muscle exhaustion and microdamage ${ }^{1,}$ ${ }^{15}$. It is possible that the fatigue, damage, and loss of flexibility in the infraspinatus and the teres minor secondary to repetitive throwing motions lead to posterior shoulder tightness. In a previous study that examined muscle activity of the upper extremities during baseball pitching using needle electromyography, the teres minor demonstrated the highest level of activity of all shoulder muscles during the deceleration phase ${ }^{9}$. Moreover, Kurokawa et al. clarified that the muscle activity ratio of the teres minor and infraspinatus during shoulder 
Posterior shoulder stretching in baseball players

274 external rotation at $90^{\circ}$ of abduction, which is necessary during the pitching motion, was 275 significantly higher than that at $0^{\circ}$ of abduction ${ }^{14}$. In other words, the throwing motion 276 requires higher intensity eccentric contraction of the teres minor than the infraspinatus; the 277 teres minor therefore tends to be more fatigued or injured, which could lead to GIRD or 278 GHAD. We suggest that the teres minor is a key muscle to consider in cases of posterior 279 shoulder tightness.

We will now discuss the effects of a 4-week stretching intervention. In both the MCS 281 and MSS groups, glenohumeral IR and HA ROM were increased. Concerning the effects of 282 a 4-week stretching intervention on ROM, glenohumeral IR and HA ROM were increased in 283 both the MCS and MSS groups. Regarding the amount of the change in the glenohumeral 284 ROM, no significant differences were found between groups. Compared with previous 285 studies on performance of stretching intervention for posterior shoulder tightness, the amount 286 of change was smaller in our study ${ }^{19,20}$. This is probably because lesser repetition or shorter 287 intervention period was performed in this study than the previous studies ${ }^{19,} 20$. Besides, 288 performing other practices is not restricted in our study, such as amount of pitching and 289 weight training for the upper body; thus, these daily practices could have affected the result 290 of this study. In the MCS group, muscle stiffness of the teres minor was decreased. In the 291 MSS group, muscle stiffness of the infraspinatus was decreased. In several previous studies 292 examining the effects of a long-term stretching intervention for posterior shoulder tightness, 293 both the cross-body and sleeper stretches were found to be effective for increasing 294 glenohumeral IR and HA ROM ${ }^{18-20}$. We investigated the effects of the MCS and MSS, which 295 are modifications of the cross-body and sleeper stretches, and determined that they are 
Posterior shoulder stretching in baseball players

296 effective for increasing glenohumeral IR and HA ROM, similar to previous studies. Moreover, 297 Akagi and Takahashi examined the effects of a 5-week stretching program for the 298 gastrocnemius using SWE and reported that muscle stiffness was decreased and ankle 299 dorsiflexion ROM was increased ${ }^{1}$. In our study, decreased muscle stiffness may be one of the 300 reasons for the increase seen in the glenohumeral ROM.

Difference was found in muscles that respond to MCS and MSS for stiffness. The previous study, which used cadavers in examining the effective position for stretching, 303 indicated that the infraspinatus could be stretched effectively by moving the shoulder into 304 internal rotation, but not by moving into horizontal adduction. The result of this study 305 supports the results of the previous study in that the stiffness of infraspinatus was decreased 306 only in the MSS group, wherein the shoulder is internally rotated. No studies quantitatively 307 examined the effective position with regard to the stretching of the teres minor. In this study, 308 the stiffness of the teres minor was decreased only in the MCS group, wherein the shoulder 309 is horizontally adducted. Another possibility is the difference in the side lying position. 310 Although both stretching methods were performed in the side lying position on the throwing 311 side, MSS was performed with the trunk rolled $30^{\circ}$ posteriorly, whereas MCS was performed 312 in the normal side lying position. Therefore, while the lateral margin of the scapula, which is 313 the region of origin of the teres minor, was compressed and fixed on the floor in MCS, the 314 infraspinatus fossa may have contacted the floor in MSS, resulting in effective stretching of 315 the infraspinatus muscle. So far, to the best of our knowledge, no previous studies examined the muscle tightness

317 before and after a period of stretching intervention in baseball players having posterior 
Posterior shoulder stretching in baseball players

318 shoulder tightness. This study showed that the MCS and MSS decreased the stiffness of the 319 teres minor and infraspinatus, respectively, and both stretching methods resulted in 320 improvement of the shoulder ROM. We think that the result of this study is useful for 321 clarifying the mechanism of posterior shoulder tightness and developing methods of 322 treatment or prevention.

\section{Limitations}

This study had several limitations. First, the number of pitches, the intensity of practice, and other stretching conditions were not controlled. Despite this, the fact that the intervention

327 showed a significant effect proves that this study is meaningful and of practical value 328 concerning the use of the MCS and MSS. Second, the glenohumeral joint capsule and 329 ligaments affecting glenohumeral ROM were not examined in this study. Most previous 330 studies have focused on the correlation between the joint capsule and posterior shoulder 331 tightness ${ }^{11-13,22,23,35}$. In these studies, plication of cadaveric posterior shoulder capsule led to 332 decreased glenohumeral IR and change in humeral head movement during glenohumeral IR 333 and HA. We did not examine these joint components; therefore, development of new methods 334 for assessing these in vivo is desired. Third, humeral torsion was not examined in this study. 335 Bailey commented that the humeral torsion did not affect shoulder stretching ${ }^{2}$; thus, we think 336 that the humeral torsion has little relation to the result in this study. Fourth, we did not classify 337 the subjects based on their symptoms such as pain; therefore, we could not determine the 338 influence of stretching on pain. Further investigation accounting for pain in a larger sample 
Posterior shoulder stretching in baseball players

339 size would be useful for assessing the effects of the MCS and MSS.

340

341 
Posterior shoulder stretching in baseball players

\section{Conclusion}

In this study, we compared glenohumeral ROM and muscle stiffness between the

344 throwing and non-throwing sides in baseball players with posterior shoulder tightness, and

345 examined the effects of a 4-week intervention using two stretching methods, the MCS and

346 MSS, on glenohumeral ROM and muscle stiffness. Baseball players with posterior shoulder

347 tightness exhibited smaller glenohumeral IR and HA ROM and greater muscle stiffness of

348 the infraspinatus and teres minor on the throwing side. The MCS and MSS are effective for

349 increasing shoulder IR and HA ROM and improving muscle stiffness of the infraspinatus and

350 teres minor. These stretching techniques can be performed by baseball players without the

351 help of a therapist, which enables them to treat or prevent posterior shoulder tightness

352 independently.

353 
Posterior shoulder stretching in baseball players

354

\section{References}

1. Akagi R, Takahashi H. Effect of a 5-week static stretching program on hardness of the gastrocnemius muscle. Scand J Med Sci Sports. 2014;24:950-957. http://dx.doi.org/10.1111/sms.12111.

2. Bailey LB, Shanley E, Hawkins R, et al. Mechanisms of Shoulder Range of Motion Deficits in Asymptomatic Baseball Players. Am J Sports Med. 2015:43:2783-93. http://dx.doi.org/ 10.1177/0363546515602446.

3. Bigliani LU, Codd TP, Connor PM, Levine WN, Littlefield MA, Hershon SJ. Shoulder motion and laxity in the professional baseball player. Am J Sports Med. 1997;25:609-613.

4. Borsa PA, Wilk KE, Jacobson JA, Scibek JS, Dover GC, Reinold MM, et al. Correlation of range of motion and glenohumeral translation in professional baseball pitchers. Am J Sports Med. 2005;33:1392-1399. http://dx.doi.org/10.1177/0363546504273490

5. Borstad JD, Dashottar A. Quantifying strain on posterior shoulder tissues during 5 simulated clinical tests: a cadaver study. J Orthop Sports Phys Ther. 2011;41:90-99. http://dx.doi.org/10.2519/jospt.2011.3357

6. Burkhart SS, Morgan CD, Kibler WB. The disabled throwing shoulder: spectrum of pathology Part I: pathoanatomy and biomechanics. Arthroscopy. 2003;19:404-420. http://dx.doi.org/10.1053/jars.2003.50128 
Posterior shoulder stretching in baseball players

374 7. Dashottar A, Costantini O, Borstad J. A comparison of range of motion change across 375 four posterior shoulder tightness measurements after external rotator fatigue. Int J $376 \quad$ Sports Phys Ther. 2014;9:498-508. NO doi

8. Digiovine NM, Jobe FW, Pink M, Perry J. An electromyographic analysis of the

9. Eby SF, Song P, Chen S, Chen Q, Greenleaf JF, An KN. Validation of shear wave elastography in skeletal muscle. J Biomech. 2013:27;46:2381-7. http://dx.doi.org/10.1016/j.jbiomech.2013.07.033.

10. Fleisig GS, Andrews JR, Dillman CJ, Escamilla RF. Kinetics of baseball pitching with implications about injury mechanisms. Am J Sports Med. 1995;23:233-239.

11. Gates JJ, Gupta A, McGarry MH, Tibone JE, Lee TQ. The effect of glenohumeral internal rotation deficit due to posterior capsular contracture on passive glenohumeral joint motion. Am J Sports Med. 2012;40:2794-2800. http://dx.doi.org/10.1177/0363546512462012

12. Harryman DT, Sidles JA, Clark JM, McQuade KJ, Gibb TD, Matsen FA. Translation of the humeral head on the glenoid with passive glenohumeral motion. J Bone Joint Surg Am. 1990;72:1334-1343.

13. Huffman GR, Tibone JE, McGarry MH, Phipps BM, Lee YS, Lee TQ. Path of glenohumeral articulation throughout the rotational range of motion in a thrower's 
Posterior shoulder stretching in baseball players

394

395

396

397

398

399

400

401

402

403

404

405

406

407

408

409

410

411

412

shoulder model. Am J Sports Med. 2006;34:1662-1669.

http://dx.doi.org/10.1177/0363546506287740

14. Kurokawa D, Sano H, Nagamoto H, Omi R, Shinozaki N, Watanuki S et al. Muscle activity pattern of the shoulder external rotators differs in adduction and abduction: an analysis using positron emission tomography. J Shoulder Elbow Surg. 2014;23:658-64. http://dx.doi.org/ 10.1016/j.jse.2013.12.021

15. Lacourpaille L, Nordez A, Hug F, Couturier A, Dibie C, Guilhem G. Time-course effect of exercise-induced muscle damage on localized muscle mechanical properties assessed using elastography. Acta Physiol (Oxf). 2014;211:135-46.

http://dx.doi.org/10.1111/apha.12272

16. Landis JR, Koch GG. The measurement of observer agreement for categorical data. Biometrics. 1977;33:159-174

17. Laudner KG, Sipes RC, Wilson JT. The acute effects of sleeper stretches on shoulder range of motion. J Athl Train. 2008;43:359-363. http://dx.doi.org/10.4085/1062-605043.4.359

18. Lintner D, Mayol M, Uzodinma O, Jones R, Labossiere D. Glenohumeral internal rotation deficits in professional pitchers enrolled in an internal rotation stretching program. Am J Sports Med. 2007;35:617-121. http://dx.doi.org/10.1177/0363546506296736 
Posterior shoulder stretching in baseball players

413

414

415

416

417

418

419

420

19. Maenhout A, Van Eessel V, Van Dyck L, Vanraes A, Cools A. Quantifying acromiohumeral distance in overhead athletes with glenohumeral internal rotation loss and the influence of a stretching program. Am J Sports Med. 2012;40:2105-2112. http://dx.doi.org/10.1177/0363546512454530

20. McClure P, Balaicuis J, Heiland D, Broersma ME, Thorndike CK, Wood A. A randomized controlled comparison of stretching procedures for posterior shoulder tightness. J Orthop Sports Phys Ther. 2007;37:108-114. http://dx.doi.org/10.2519/jospt.2007.2337

21. Moore SD, Laudner KG, McLoda TA, Shaffer MA. The immediate effects of muscle energy technique on posterior shoulder tightness: a randomized controlled trial. J Orthop Sports Phys Ther. 2011;41:400-407.http://dx.doi.org/ 10.2519/jospt.2011.3292

22. Muraki T, Yamamoto N, Zhao KD, et al. Effect of posteroinferior capsule tightness on contact pressure and area beneath the coracoacromial arch during pitching motion. Am J Sports Med. 2010;38:600-607. http://dx.doi.org/10.1177/0363546509350074

23. Muraki T, Yamamoto N, Zhao KD, Sperling JW, Steinmann SP, Cofield RH, et al. Effects of posterior capsule tightness on subacromial contact behavior during shoulder motions. J Shoulder Elbow Surg. 2012;21:1160-1167. http://dx.doi.org/ 10.1016/j.jse.2011.08.042

24. Myers JB, Laudner KG, Pasquale MR, Bradley JP, Lephart SM. Glenohumeral range of motion deficits and posterior shoulder tightness in throwers with pathologic 
Posterior shoulder stretching in baseball players

433

434

435

436

437

438

439

440

441

442

443

444

445

446

447

448

449

450

internal impingement. Am J Sports Med. 2006;34:385-391.

http://dx.doi.org/10.1177/0363546505281804

25. Myers JB, Oyama S, Wassinger CA, Ricci RD, Abt JP, Conley KM, et al. Reliability, precision, accuracy, and validity of posterior shoulder tightness assessment in overhead athletes. Am J Sports Med. 2007;35:1922-1930.

http://dx.doi.org/10.1177/0363546507304142

26. Nakamura M, Ikezoe T, Kobayashi T, et al. Acute effects of static stretching on muscle hardness of the medial gastrocnemius muscle belly in humans: an ultrasonic shear-wave elastography study. Ultrasound Med Biol. 2014;40:1991-1997. http://dx.doi.org/10.1016/j.ultrasmedbio.2014.03.024

27. Oyama S, Goerger CP, Goerger BM, Myers JB. Effects of non-assisted posterior shoulder stretches on shoulder range of motion among collegiate baseball players. Athletic Training and Sports Health Care. 2010;2;163-170. http://dx.doi.org/10.3928/19425864-20100524-01

28. Oyama S, Myers JB, Blackburn JT, Colman EC. Changes in infraspinatus crosssectional area and shoulder range of motion with repetitive eccentric external rotator contraction. Clin Biomech (Bristol, Avon). 2011;26:130-135.

http://dx.doi.org/10.1016/j.clinbiomech.2010.09.018 
Posterior shoulder stretching in baseball players

451

452

453

454

455

456

457

458

459

460

461

462

463

464

465

466

467

468

469

470

29. Park KN, Kwon OY, Weon JH, Choung SD, Kim SH. Comparison of the effects of local cryotherapy and passive cross-body stretch on extensibility in subjects with posterior shoulder tightness. J Sports Sci Med. 2014; 20;13:84-90. No doi.

30. Poser A, Casonato O. Posterior glenohumeral stiffness: capsular or muscular problem? A case report. Man Ther. 2008;13:165-170.

http://dx.doi.org/10.1016/j.math.2007.07.002

31. Reinold MM, Wilk KE, Macrina LC, Sheheane C, Dun S, Fleisig GS, et al. Changes in shoulder and elbow passive range of motion after pitching in professional baseball players. Am J Sports Med. 2008;36:523-527.

http://dx.doi.org/10.1177/0363546507308935

32. Ruotolo C, Price E, Panchal A. Loss of total arc of motion in collegiate baseball players. J Shoulder Elbow Surg. 2006;15:67-71.

http://dx.doi.org/10.1016/j.jse.2005.05.006

33. Salamh PA, Kolber MJ, Hanney WJ. Effect of scapular stabilization during horizontal adduction stretching on passive internal rotation and posterior shoulder tightness in young women volleyball athletes: a randomized controlled trial. Arch Phys Med Rehabil. 2015;96:349-356. http://dx.doi.org/10.1016/j.apmr.2014.09.038.

34. Scher S, Anderson K, Weber N, Bajorek J, Rand K, Bey MJ. Associations among hip and shoulder range of motion and shoulder injury in professional baseball players. $\mathrm{J}$ Athl Train. 2010;45:191-197. http://dx.doi.org/10.4085/1062-6050-45.2.191 
Posterior shoulder stretching in baseball players

471

472

473

474

475

476

477

478

479

480

481

482

483

484

485

486

487

488

489

490

35. Shanley E, Rauh MJ, Michener LA, Ellenbecker TS, Garrison JC, Thigpen CA. Shoulder range of motion measures as risk factors for shoulder and elbow injuries in high school softball and baseball players. Am J Sports Med. 2011;39:1997-2006. http://dx.doi.org/10.1177/0363546511408876

36. Thomas SJ, Swanik CB, Higginson JS, Kaminski TW, Swanik KA, Bartolozzi AR, et al. A bilateral comparison of posterior capsule thickness and its correlation with glenohumeral range of motion and scapular upward rotation in collegiate baseball players. J Shoulder Elbow Surg. 2011;20:708-716.

http://dx.doi.org/10.1016/j.jse.2010.08.031

37. Wilk KE, Macrina LC, Arrigo C. Passive range of motion characteristics in the overhead baseball pitcher and their implications for rehabilitation. Clin Orthop Relat Res. 2012;470:1586-1594. http://dx.doi.org/10.1007/s11999-012-2265-z

38. Wilk KE, Hooks TR, Macrina LC. The modified sleeper stretch and modified crossbody stretch to increase shoulder internal rotation range of motion in the overhead throwing athlete. J Orthop Sports Phys Ther. 2013;43:891-894.

http://dx.doi.org/10.2519/jospt.2013.4990

39. Wilk KE, Macrina LC, Fleisig GS, Porterfield R, Simpson CD 2nd, Harker P, et al. Correlation of glenohumeral internal rotation deficit and total rotational motion to shoulder injuries in professional baseball pitchers. Am J Sports Med. 2011;39:329335. http://dx.doi.org/10.1177/0363546510384223 
Posterior shoulder stretching in baseball players

491

492

493

494

495

496

497

498

499

500

501

502
40. Yanagisawa O, Niitsu M, Takahashi H, Itai Y. Magnetic resonance imaging of the rotator cuff muscles after baseball pitching. J Sports Med Phys Fitness. 2003;43:493499. No doi

41. Yang JL, Chen SY, Hsieh CL, Lin JJ. Effects and predictors of shoulder muscle massage for patients with posterior shoulder tightness. BMC Musculoskelet Disord. 2012;13:46. http://dx.doi.org/10.1186/1471-2474-13-46

42. Yoshitake Y, Takai Y, Kanehisa H, Shinohara M. Muscle shear modulus measured with ultrasound shear-wave elastography across a wide range of contraction intensity. Muscle Nerve. 2014;50:103-13. http://dx.doi.org/10.1002/mus.24104. 
Posterior shoulder stretching in baseball players

503

504

505

506

507

508

509

510

511

512

513

514

515

516

517 glenohumeral horizontal adduction.

Table 1
Intra-class Correlation Coefficient $[\operatorname{ICC}(1,2)]$ Values (intra-rater)

\begin{tabular}{|c|c|c|c|}
\hline & & $\operatorname{ICC}(1,2)$ & SEM \\
\hline \multirow{3}{*}{ ROM } & IR & 0.88 & 2.37 \\
\hline & $\mathrm{ER}$ & 0.93 & 3.18 \\
\hline & $\mathrm{HA}$ & 0.92 & 4.49 \\
\hline \multirow{3}{*}{$\begin{array}{l}\text { Muscle Stiffness } \\
\text { 2nd IR Position }\end{array}$} & Infraspinatus & 0.73 & 1.39 \\
\hline & Teres Minor & 0.88 & 1.50 \\
\hline & Posterior Deltoid & 0.77 & 1.26 \\
\hline \multirow{3}{*}{$\begin{array}{c}\text { Muscle Stiffness } \\
\text { HA Position }\end{array}$} & Infraspinatus & 0.75 & 3.67 \\
\hline & Teres Minor & 0.79 & 3.47 \\
\hline & Posterior Deltoid & 0.84 & 2.86 \\
\hline
\end{tabular}

518

519

520 
Posterior shoulder stretching in baseball players

521

522

\begin{tabular}{|c|c|c|c|}
\hline & & MCS (12) & MSS (11) \\
\hline Age (year) & & $21.4 \pm 1.2$ & $20.3 \pm 0.9$ \\
\hline Height (cm) & & $173.0 \pm 4.0$ & $171.7 \pm 6.9$ \\
\hline Mass (kg) & & $70.4 \pm 3.0$ & $68.6 \pm 6.0$ \\
\hline \multirow{4}{*}{ ROM () } & IR & $49 \pm 6$ & $53 \pm 6$ \\
\hline & $\mathrm{ER}$ & $118 \pm 9$ & $116 \pm 8$ \\
\hline & TOTAL & $168 \pm 9$ & $169 \pm 7$ \\
\hline & $\mathrm{HA}$ & $81 \pm 11$ & $86 \pm 9$ \\
\hline \multirow{4}{*}{$\begin{array}{l}\text { Muscle Stiffness } \\
\text { 2nd IR Position (kPa) }\end{array}$} & Infraspinatus & $9.8 \pm 2.4$ & $10.0 \pm 2.8$ \\
\hline & Teres Minor & $15.5 \pm 4.5$ & $13.5 \pm 2.9$ \\
\hline & Posterior & \multirow[b]{2}{*}{$10.1 \pm 5.0$} & \multirow[b]{2}{*}{$8.3 \pm 2.3$} \\
\hline & Deltoid & & \\
\hline \multirow{4}{*}{$\begin{array}{l}\text { Muscle Stiffness } \\
\text { HA Position (kPa) }\end{array}$} & Infraspinatus & $12.6 \pm 4.5$ & $11.3 \pm 5.7$ \\
\hline & Teres Minor & $19.6 \pm 5.3$ & $17.5 \pm 5.1$ \\
\hline & Posterior & \multirow[b]{2}{*}{$30.5 \pm 6.5$} & \multirow[b]{2}{*}{$31.4 \pm 8.5$} \\
\hline & Deltoid & & \\
\hline
\end{tabular}

Table 2. Baseline Characteristics of Participants

524 Abbreviations: MCS; modified cross-body stretch, MSS; modified sleeper

525 stretch, ROM; range of motion, IR; glenohumeral internal rotation, ER; 
Posterior shoulder stretching in baseball players

526 glenohumeral external rotation, TOTAL; total glenohumeral rotation, HA;

527 glenohumeral horizontal adduction,

528 Values are presented as mean $\pm \mathrm{SD}$.

529 At baseline, there was no significant difference between the two groups.

530

531 
Posterior shoulder stretching in baseball players

532

533

534

535

\section{Table3}

Comparison of Dominant and Non-Dominant Shoulders

(baseline differences)

535

$\operatorname{ROM}\left({ }^{\circ}\right)$

Throwing Non-Throwing $\mathrm{P}$

\begin{tabular}{ccccc} 
& & Side & Side & value \\
\hline & IR & $51 \pm 7$ & $66 \pm 7^{*}$ & $<.001$ \\
ROM $\left(^{\circ}\right)$ & TOTAL & $118 \pm 8$ & $106 \pm 10$ & $<.001$ \\
& HA & $83 \pm 10$ & $109 \pm 11^{*}$ & $<.001$ \\
\hline \multirow{2}{*}{ Muscle Stiffness } & Infraspinatus & $9.8 \pm 2.6^{*}$ & $8.1 \pm 2.3$ & $<.001$ \\
& Teres Minor & $14.3 \pm 4.0^{*}$ & $10.0 \pm 3.1$ & $<.001$ \\
2nd IR Position (kPa) & Posterior Deltoid & $9.2 \pm 3.9$ & $8.4 \pm 2.6$ & 0.16 \\
\hline \multirow{2}{*}{ Muscle Stiffness } & Infraspinatus & $11.1 \pm 4.2$ & $10.2 \pm 5.0$ & 0.16 \\
& Teres Minor & $18.5 \pm 5.1^{*}$ & $14.3 \pm 4.5$ & $<.001$ \\
HA Position (kPa) & Posterior Deltoid & $31.1 \pm 7.3$ & $29.1 \pm 7.1$ & 0.12 \\
\hline
\end{tabular}

\section{6}

537 Abbreviations: ROM; range of motion, IR; glenohumeral internal rotation, ER;

538 glenohumeral external rotation, TOTAL; total glenohumeral rotation, HA;

539 glenohumeral horizontal adduction.

$540 \quad$ Values are presented as mean $\pm \mathrm{SD}$.

$541 *$ Significant differences between the throwing and non-throwing sides 
Posterior shoulder stretching in baseball players

544

545

546

\begin{tabular}{cccccc}
\hline & & Pre & Post & Amount of & \\
& Group & Stretching $\left(^{\circ}\right)$ & Stretching $\left(^{\circ}\right)$ & Change $\left.{ }^{\circ}\right)$ & P value \\
\hline IR & MCS & $49 \pm 6$ & $57 \pm 7^{* *}$ & $8 \pm 5$ & $<.001$ \\
& MSS & $53 \pm 6$ & $61 \pm 6^{* *}$ & $8 \pm 4$ & $<.001$ \\
\hline ER & MCS & $118 \pm 9$ & $122 \pm 8$ & $3 \pm 8$ & 0.19 \\
\hline TOTAL & MSS & $116 \pm 8$ & $119 \pm 8$ & $3 \pm 8$ & 0.22 \\
\hline HA & MSS & $169 \pm 7$ & $180 \pm 10^{*}$ & $11 \pm 11$ & $<.001$ \\
& MCS & $81 \pm 11$ & $86 \pm 9^{* *}$ & $6 \pm 5$ & $<.001$ \\
\hline
\end{tabular}

547

548 Abbreviations: MCS; modified cross-body stretch, MSS; modified sleeper

549 stretch, ROM; range of motion, IR; glenohumeral internal rotation, ER;

550 glenohumeral external rotation, TOTAL; total glenohumeral rotation, HA;

551 glenohumeral horizontal adduction.

$552 \quad$ Values are presented as mean $\pm \mathrm{SD}$.

553 * Significant post-stretching changes in ROM compared to pre-stretching

$554 \quad(\mathrm{P}<.05)$

$555 * *$ Significant post-stretching changes in ROM compared to pre-stretching 
Posterior shoulder stretching in baseball players

$556 \quad(\mathrm{P}<.01)$

557

558

559

560 
Posterior shoulder stretching in baseball players

561

562

563

\section{Table 5}

Effects of a 4-Week Stretching Program on Muscle Stiffness

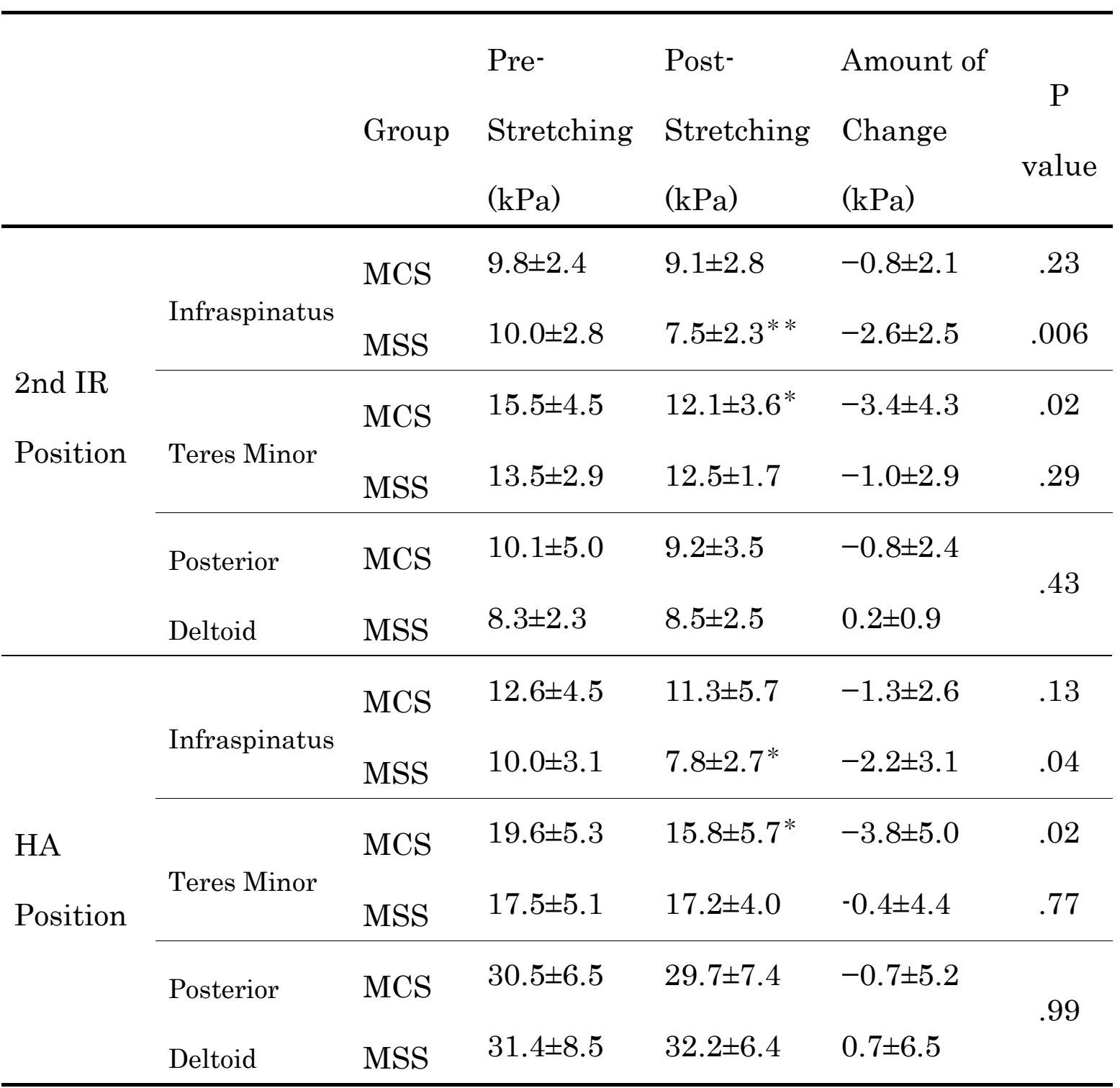

565 Abbreviations: MCS; modified cross-body stretch, MSS; modified sleeper

566 stretch, IR; glenohumeral internal rotation, HA; glenohumeral horizontal 567 adduction. 
Posterior shoulder stretching in baseball players

$568 \quad$ Values are presented as mean $\pm \mathrm{SD}$.

569 * Significant post-stretching changes in muscle stiffness compared to pre-

$570 \quad$ stretching $(\mathrm{P}<.05)$

$571 * *$ Significant post=stretching changes in muscle stiffness compared to pre-

$572 \quad$ stretching $(\mathrm{P}<.01)$

573

574

575 
Posterior shoulder stretching in baseball players

576 Fig.1. Assessment of Shoulder Muscle Stiffness Using SWE

577 A color-coded box showing the shear elastic modulus superimposed on the B-

578 mode ultrasound image; the circular region of interest (ROI) was set near the

579 central part of the muscle, and we used the average ROI for analysis.

580 Abbreviations: ISP; infraspinatus, TM; teres minor, PD; posterior deltoid

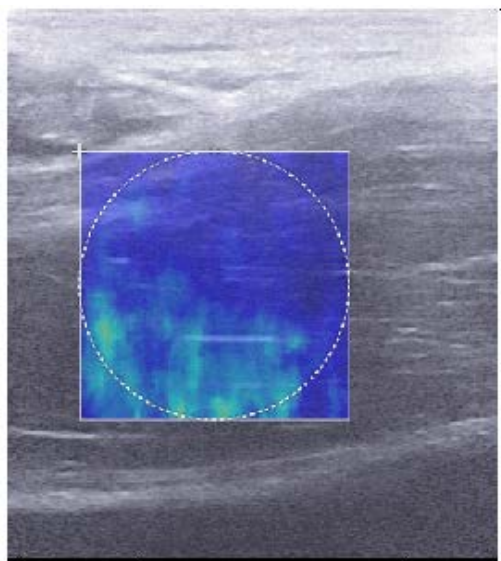

ISP

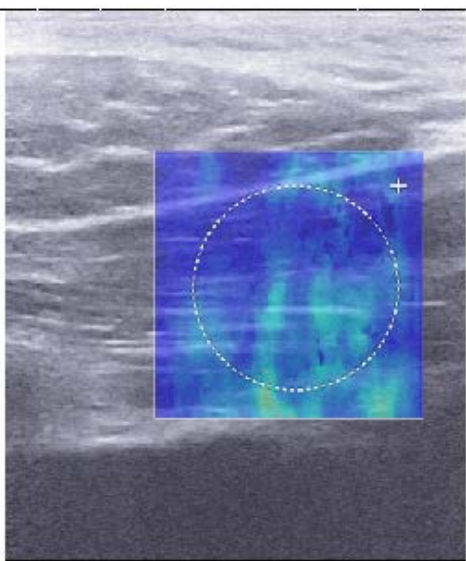

TM

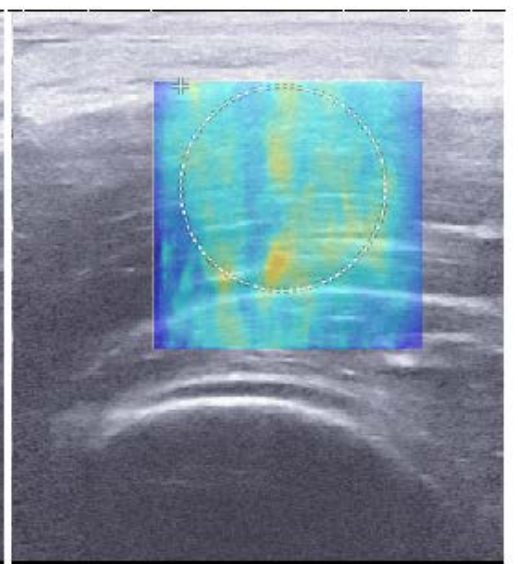

PD

582

583 
Posterior shoulder stretching in baseball players

\section{Fig.2. Probe Placement for Each Muscle}

585 The probe placement is shown, with the subject seated and shoulder and elbow

586 flexed to $90^{\circ}$. The ISP is measured at the midpoint of the spine of the scapula

587 and its inferior angle, and the probe is placed parallel to the ISP. The TM is

588 measured near the midpoint of the inferior angle of the scapula and greater

589 tubercle, where the TM is identified with the probe vertical to the TM, and

590 then the probe is placed parallel to the TM. The PD is measured at $4 \mathrm{~cm}$ below

591 the posterior acromion, and the probe is placed parallel to the PD.

592 Abbreviations: ISP; infraspinatus, TM; teres minor, PD; posterior deltoid, SS;

593 spine of the scapula, IA; inferior angle of scapula, GT; greater tubercle. 
Posterior shoulder stretching in baseball players

595

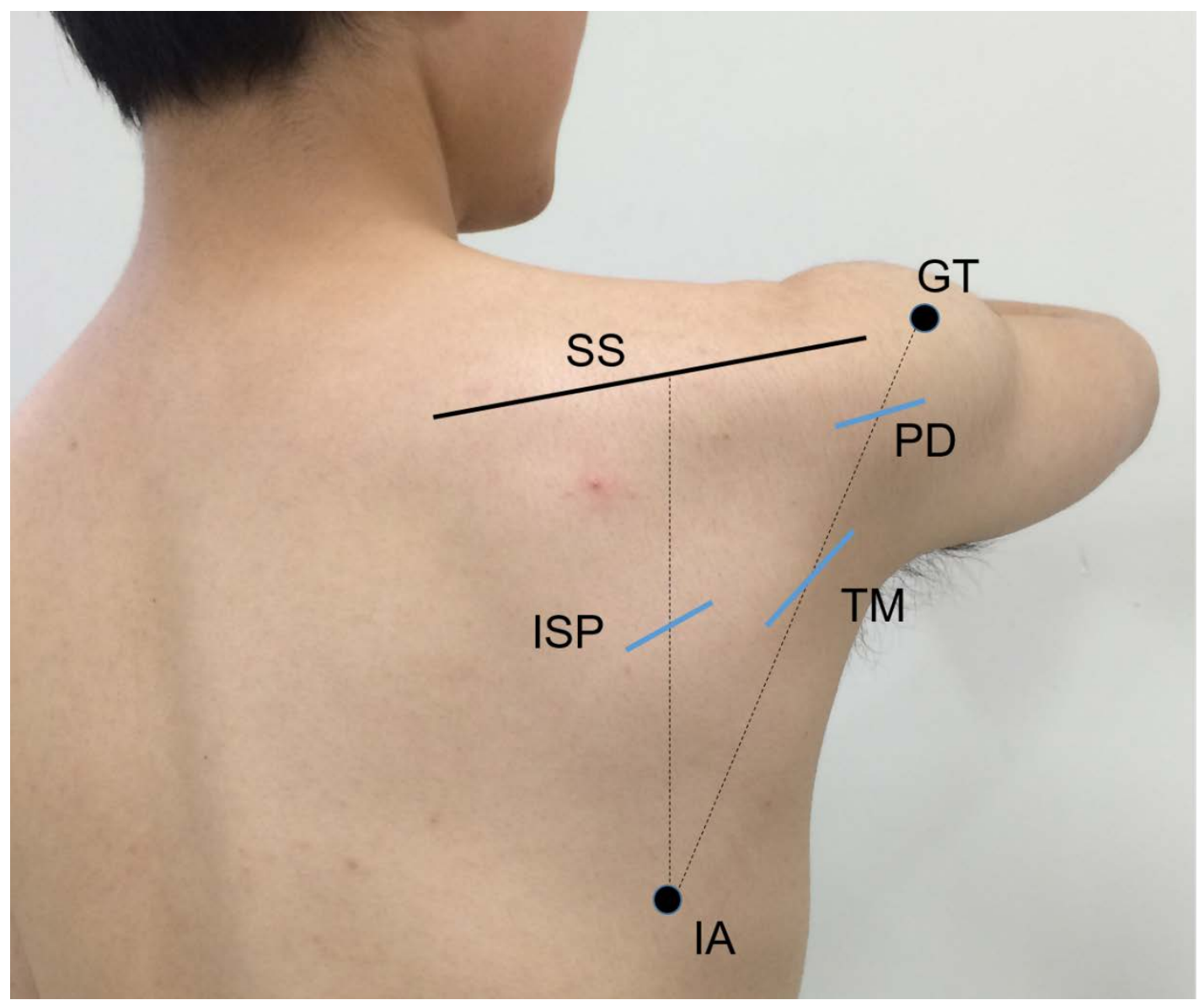

596

597 
Posterior shoulder stretching in baseball players

598 Fig.3. Two Stretching Methods - MCS and MSS

599 In this study, we used the MCS and the MSS.

600

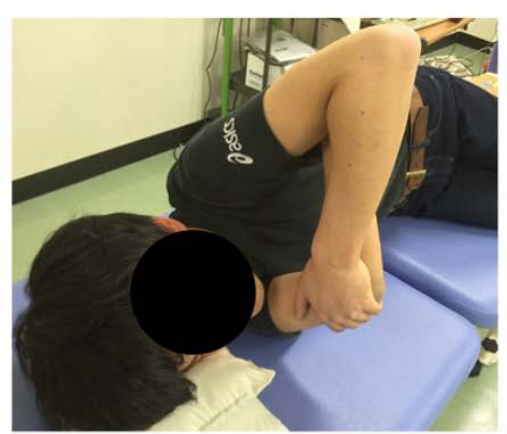

modified cross-body stretch (MCS)

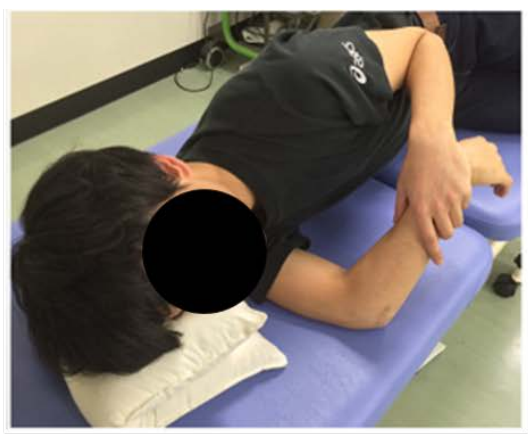

modified sleeper stretch (MSS)

601

602 
Posterior shoulder stretching in baseball players

Fig.4. Flow Diagram Representing Enrollment, Allocation, Procedures, and

604 Analysis.

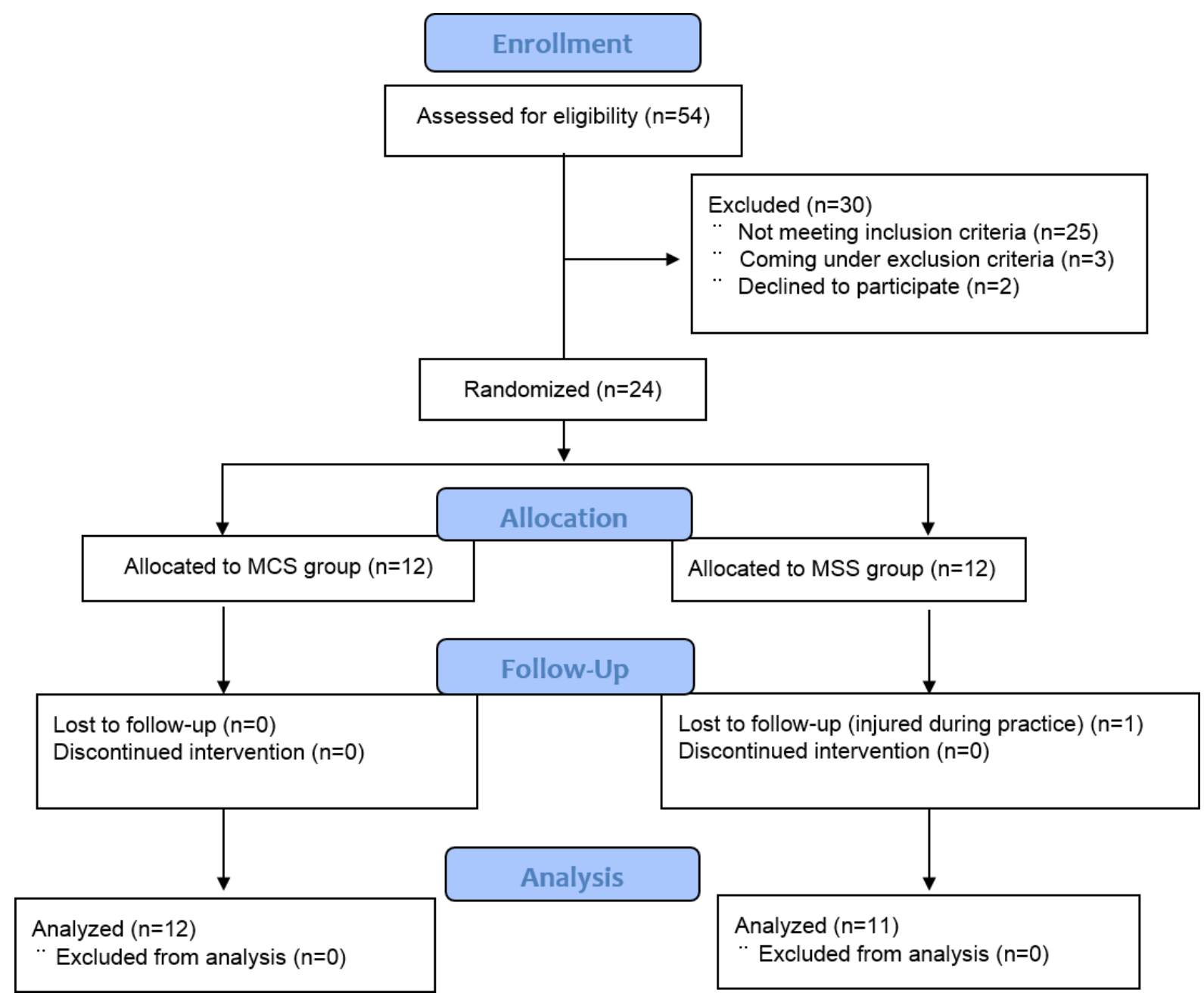

605

606

607 